\title{
Development of LYSO detector modules for a charge-particle EDM polarimeter
}

\section{Shergelashvili*1, D. Mchedlishvili ${ }^{1,2}$, F. Müller ${ }^{3,4}$, I. Keshelashvili ${ }^{4}$ for the JEDI collaboration)}

${ }^{1}$ SMART I EDM_Lab, Tbilisi State University, 0179 Tbilisi, Georgia

${ }^{2}$ High-Energy Physics Institute, Tbilisi State University, 0186 Tbilisi, Georgia

${ }^{3}$ Physics Institute III B, RWTH Aachen, Otto-Blumenthal-Strasse, 52074 Aachen, Germany

${ }^{4}$ Institute of Nuclear Physics, Forschungszentrum Jülich, 52425 Jülich, Germany

E-mail: shergelashvili.dito@gmail.com

The JEDI (Jülich Electric Dipole moment Investigations) collaboration carries out preparations, $\mathrm{R} \& \mathrm{D}$ and a first proof-of-principle measurement for the challenging project to measure permanent electric dipole moments of charged particles using a storage ring. In the long term, the experiments are targeted at the construction of a novel storage ring including a new polarimeter based on inorganic LYSO crystals and low voltage Silicon Photomultipliers (SiPMs). In order to find the best material and assembling configurations, more than 50 modules have been assembled in different set-ups so far. Modules have first been tested in the laboratory with internal and external radiation sources and subsequently, they have been examined under experimental conditions employing accelerator beams. We have performed five test beam times at several different beam energies using the new DAQ system, which was optimized for the new polarimeter. The results of these measurements and the accumulated experience of the module production will be presented.

23rd International Spin Physics Symposium - SPIN2018 -

10-14 September, 2018

Ferrara, Italy

${ }^{*}$ Speaker. 


\section{Introduction}

The JEDI collaboration was formed to exploit COSY (COoler SYnchrotron) facility and make all necessary investigation toward the design of a dedicated storage ring which aims to search Electric Dipole Moment (srEMD) of charged particles [1]. The key challenge for srEDM is the provision of a sensitive and efficient method to determine the tiny change of the beam polarization direction. The new concept of the dedicated polarimetry (target, detection system, data acquisition), will allow to push the EDM sensitivity level by orders of magnitude in a future storage ring $[3,4]$.

According to the requirement (precision, efficiency, stability, no electric/magnetic field, compact) the polarimetry development consists of two main parts: target and detector system constructions [5]. The main Current experiments are focused on the development of the calorimeter part of the detector. The material for the new calorimeter is LYSO (Lutetium-yttrium oxy orthosilicate) scintillator crystal because of its special properties, such as high density, high light output, short decay time and good stability [2]. Scintillation light readout is made by a SiPM array (Silicon PhotoMultiplier), which generates an analog signal. This signal needs to be digitized for data recording and further analysis. The Following flow chart shows the basic working principle of the polarimeter: Particle deposits energy in the crystal and proportional numbers of scintillation photons are emitted. The SiPM registers photons and generates the analog signal. The output of Multiple modules are sampled by the fast ADCs and the digital information is distributed via $10 \mathrm{Gbit} / \mathrm{s}$ network to the server computer. The server computer records and sends the data stream to the online analysis. High stability and low noise voltage supply is designed specifically for the SiPM. Supply voltage range is adjustable within 25-31 volts and can be set according to the experiment needs.

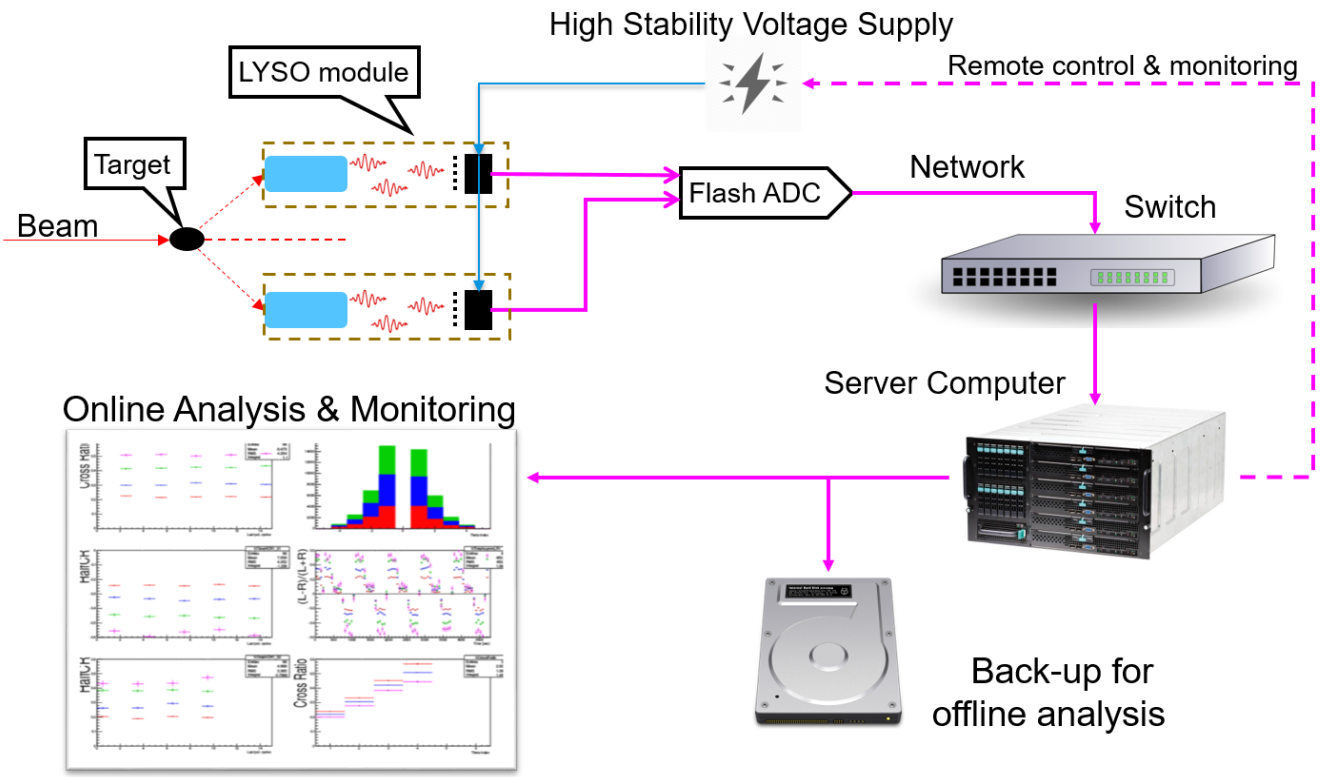

Figure 1: Polarimetry structure 


\section{LYSO module construction and inspection with portable DAQ}

The LYSO modules for the JEDI polarimeter were assembled in Forschungszentrum Jülich. The multiple tests ware performed before the final version of the module was established. Modules consist of two main parts: an LYSO crystal, manufactured according to our requirements, and a SiPM sensor chip. The crystal is $8 \mathrm{~cm}$ long with transverse sizes of $3 \times 3 \mathrm{~cm}^{2}$. This length is sufficient to fully stop deuterons with up to $300 \mathrm{MeV}$ kinetic energy. The specific Brag peak is located at about $6 \mathrm{~cm}$ deep for the $270 \mathrm{MeV}$ deuterons (experimental result).

The crystals are read out by Silicon Photo-multiplier arrays with $3 \times 3 \mathrm{~cm}^{2}$-sized boards. Each board consists of 64 cells of SensL J-Series chips with $14 \mathrm{~K}-20 \mu \mathrm{m}$ pixels. In total 900k pixels are responsible for detecting photons from each LYSO crystal [6]. The SiPM board has a surfacemounted connector and can be easily attached to the module PCB. This PCB is presented with passive components and manages power input and signal out rails.
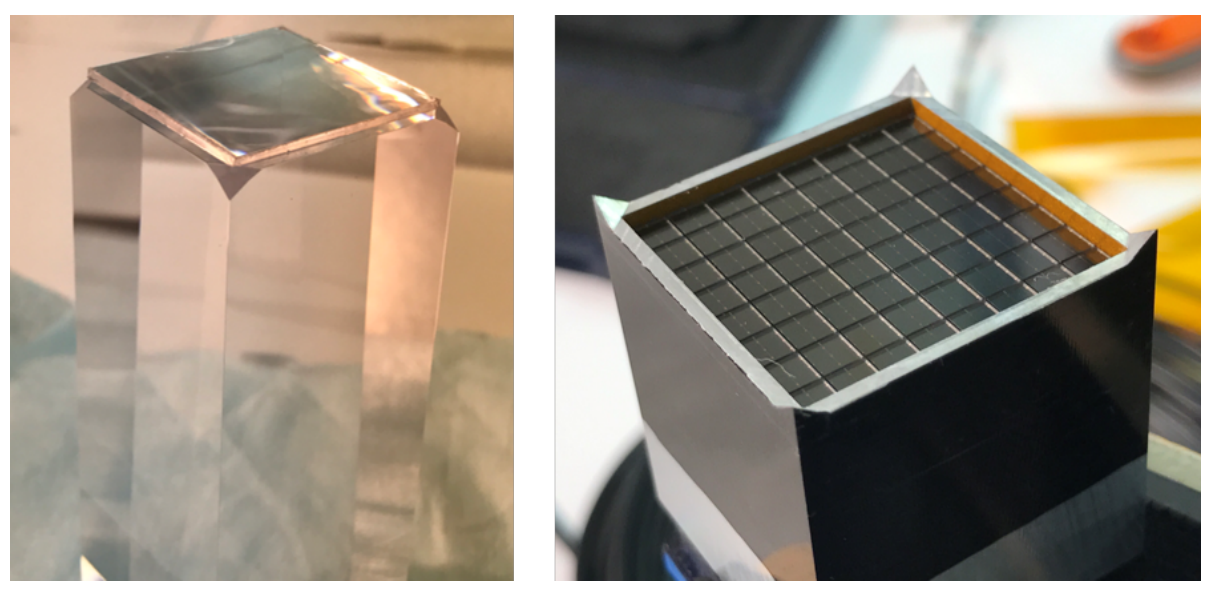

Figure 2: LYSO Crystal (left) and SiMP Board (right)

The tight connection between the LYSO crystal and the SiPM board is made by using special parts from different materials which create a whole module body. The module integrity is guaranteed by 3D printed plastic materials, aluminum housing, springs and foils. The SiPM and the crystal surfaces are separated by a $1 \mathrm{~mm}$ thick silicon pad to improves the optical contact also soften the mechanical strain between them. Besides, the pad also protects the SiPM pixels from the direct internal radiation of the LYSO crystal. Room light tightness is very important to avoid wrong measurements which are realized with $50 \mu \mathrm{m}$ tedlar foil. The tedlar is wrapped around the teflon foil which is responsible for light reflection and leads to higher light output and hence, larger signal. The mechanical fixation and construction stability are ensured by a kapton foil. The amount of building materials around the module is minimized. This simplifies the module installation with different configurations and introduces minimal energy loss for the incident particles (less than 1\%) through the foils. 
The module quality control and calibration are done using the special test bench. For the inspection, two different radioactive sources ${ }^{60} \mathrm{Co},{ }^{22} \mathrm{Na}$ are used. The test bench consists of portable DAQ system and SiPM voltage supply. The DAQ system is based on the Raspberry Pi computer and the Red Pitaya board. The DAQ software was developed using the CERN ROOT libraries. The test procedure includes several steps. The first test checks the powering of the modules. It implies the tuning of the supply voltage for each module to achieve the best performance (high gain, good signal to noise ratio). The electrical characteristics of SiPM arrays and the connector board (including wiring and passive components) are checked by the micro ammeter. The ammeter measures the leakage current in the bypass capacitors (must be $<1 \mu \mathrm{A}$ ) and the "dark current" through the SiPMs (must be $<10 \mu \mathrm{A}$ ) at the supply voltage of $30.000 \mathrm{~V}$. In the next step a real measurement takes place using DAQ. Before using the radioactive sources, light tightness measurement is performed in order to ensure the wrapping quality. The next steps aim to check LYSO module performance together with light-sensitive parts. For that, two different ${ }^{60} \mathrm{Co}$ and ${ }^{22} \mathrm{Na}$ sources are used separately. This test examines LYSO module performance (linearity, resolution) at low energies and is followed by further tests at the COSY experimental area.

\section{JEDI polarimeter DAQ System}

According to the dedicated polarimeter concept, a multi-channel, fast ADC is needed. The SIS3316 FADC module from "Struck innovative systeme" is well-suited with these requirements. It has 16 individual channels with $250 \mathrm{MSamples/s}$ sampling frequency and 14-bit resolution. The modules can be synchronized to work in a chained configuration, allowing for parallel data acquisition. The FADC module includes 5 FPGA chips and has $2 \mathrm{~Gb}$ total internal memory[7]. The modules have various triggering modes and allow for configuration of triggering conditions. The SIS3316 module first fills internal memories and, after reaching the memory threshold, sends bunched data thought the 10 Gbit network to the server computer. The whole data flow chain is presented on the following figure:

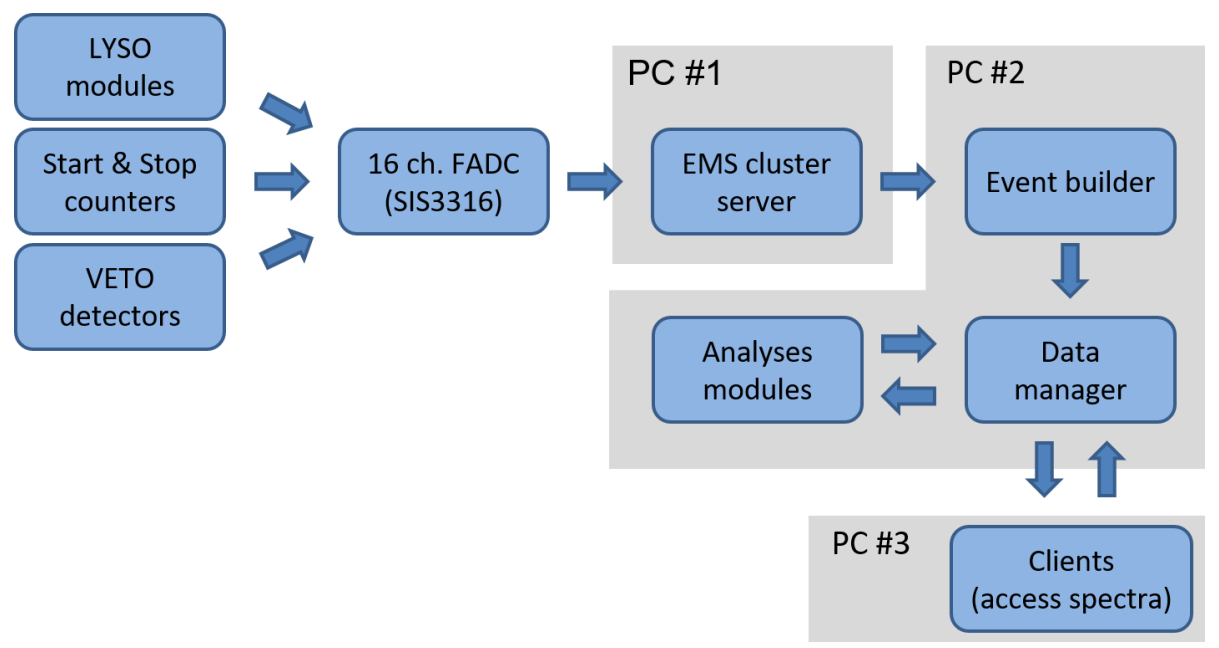

Figure 3: Polarimeter DAQ data flow chart 
The server computer also receives the status information from the whole experimental area and shares it to the second computer. This computer is the main core for data analysis and communication with client/user. Software packages running on the $2^{\text {nd }}$ computer is based on CERN ROOT libraries. The DAQ software is able to record the data and simultaneously do an online analysis for a client.

\section{Results}

Development of the JEDI polarimeter requires complex tests in order to find a best configuration for the desired measurement. Hardware and software parts can be checked in the laboratory environment without beam. But it's important to check detector performance with polarized/unpolarized beams as well. For the experiment special arrangement was prepared. The deuteron beam with energy $300 \mathrm{MeV}$ was directed to the $5 \mathrm{~mm}$ thick carbon block target. Each arm of the detector consisted of 12 LYSO modules ( 3 peace in the row and 4 peace in the column). The front area of these 12 modules assembly was covered by the plastic scintillator in such a way, that particles flying from the target first passed the scintillator and then be stopped in the LYSO crystal. The following 2D histogram shows energy deposition in the plastic scintillator (OY axis) versus LYSO modules (OX axis):

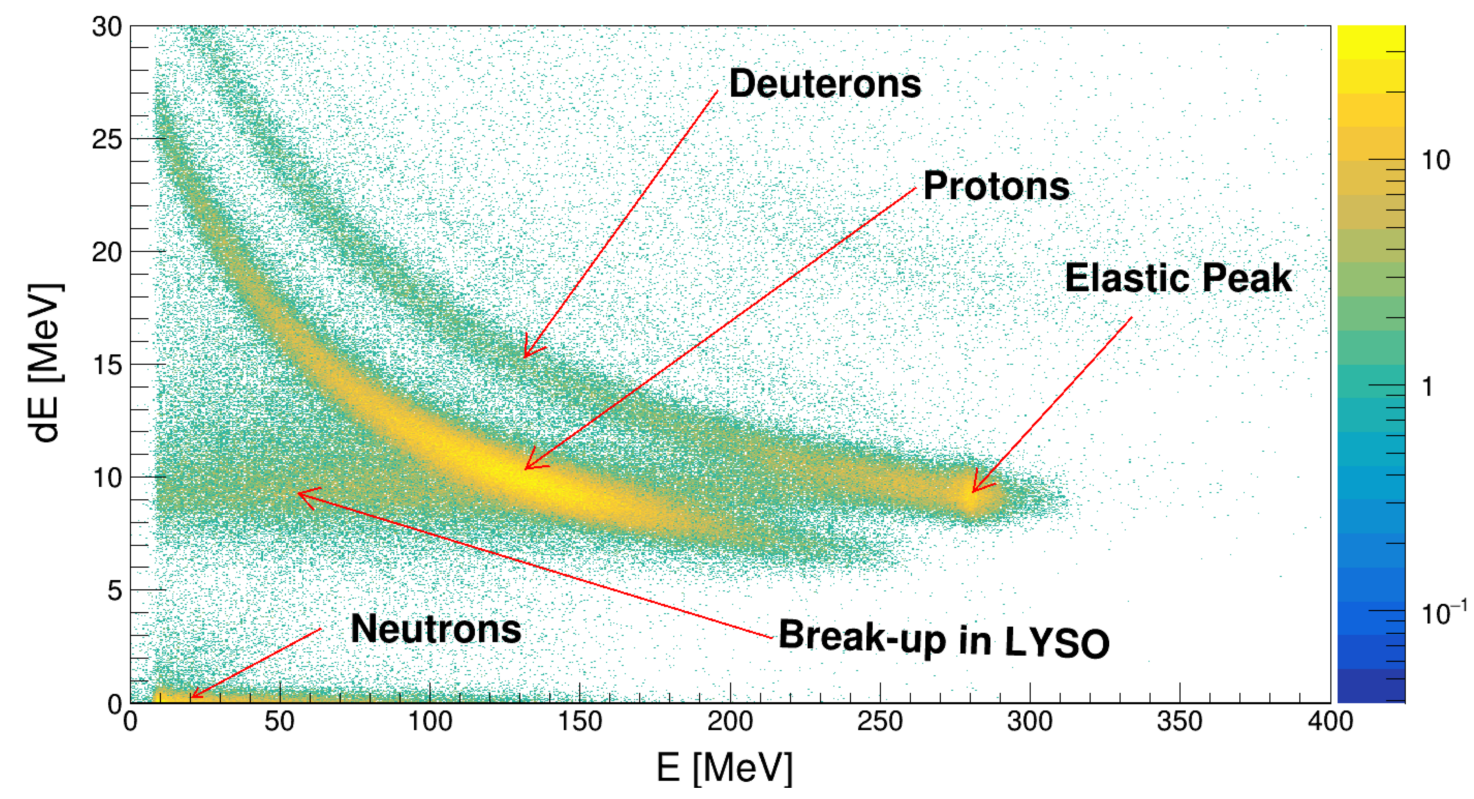

Figure 4: $d E$ vs. E at $300 \mathrm{MeV}$ Deuteron beam and carbon target

The histogram shows various reaction areas: The first, upper band corresponds to $\mathrm{dC}$ while the second one describes break-up protons in the carbon target. The upper horizontal band on the fig: 4, extending up to the elastic peak, corresponds to deuterons which break-up in the LYSO. According to the data, break-up protons from deuteron break-up on Carbon events are much more frequent than elastic $\mathrm{dC}$ events. This encouraged us to check how useful these events could be for the asymmetry measurement. For this purpose the OY axis of fig: 4 was transformed in a way to 
simplify the selection of different processes. The transformed distribution is shown on fig: 5(left). These events are divided into 4 segments and individual asymmetries are presented on fig: 5 (right).
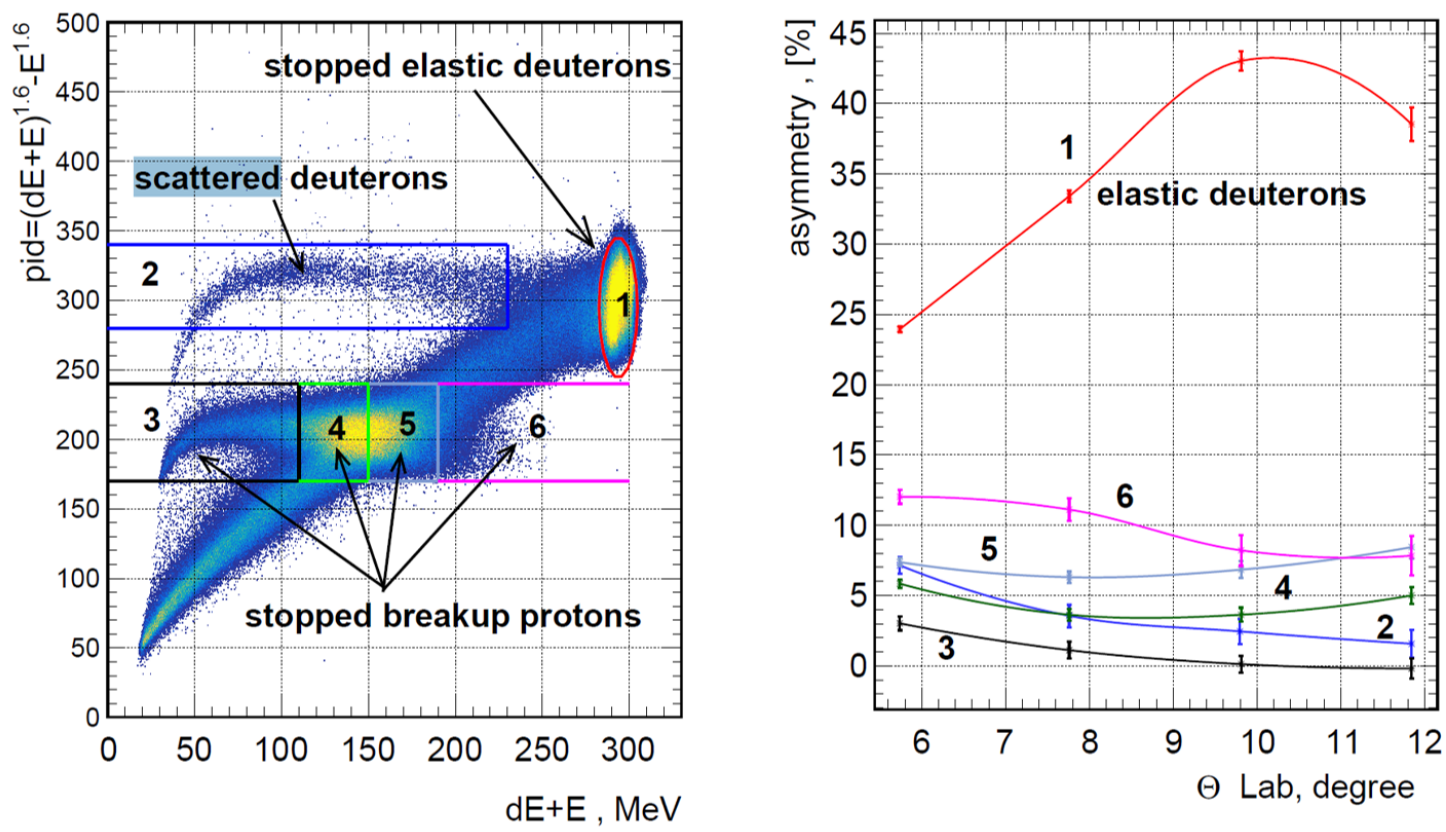

Figure 5: Particle Identification (left), Asymmetry (right)

According to the analysis results, break-up reaction shows some degree of polarisation sensitivity. However, the elastic $\mathrm{dC}$ reaction still remains the best candidate for asymmetry measurements at this energy and at low angles. The break-up process asymmetry, as observed on fig: 5 (right), tends to increase at larger angles, that are already out of our detector acceptance. These higher angles might be further investigated in the future.

\section{Acknowledgements}

This work has been financially supported by the Forschungszentrum JÃijlich via COSY-FFE, by the European Research Council Advanced-Grant (srEDM, No. 694340) and by the grant from the Shota Rustaveli National Science Foundation of the Republic of Georgia (SRNSF Grant agreement 04/01, "Search for Electric Dipole Moments using Storage Rings (srEDM)". 


\section{References}

[1] JEDI Collaboration, http://collaborations.fz-juelich.de/ikp/jedi

[2] C. Melcher and J. Schweitzer, IEEE Trans. Nucl. Sci. 39, 502 (1992)

[3] V. Anastassopoulos et al., "A Storage Ring Experiment to Detect a Proton Electric Dipole Moment", arXiv:1502.04317 [physics.acc-ph] (2015)

[4] J. Pretz, "Measurement of Permanent Electric Dipole Moments of Charged Hadrons in Storage Rings", Hyperfine Interact., vol. 214, no. 1-3, pp. 111-117, (2013)

[5] I. Keshelashvili "Towards JEDI Polarimetry", XVIth International Workshop in Polarized Sources, Targets, and Polarimetry, PoS(PSTP2015)026

[6] https://www.sensl.com/downloads/ds/TN\%20-\%20Intro\%20to\%20SPM\%20Tech.pdf

[7] http://www.struck.de/sis3316.html 